\title{
An Analysis of a Superconvergence Result for a Singularly Perturbed Boundary Value Problem*
}

\author{
By Eugene O'Riordan and Martin Stynes
}

\begin{abstract}
We give a new proof that the El-Mistikawy and Werle finite-difference scheme is uniformly second-order accurate for a nonselfadjoint singularly perturbed boundary value problem. To do this, we use exponential finite elements and a discretized Green's function. The proof is direct, gives the nodal errors explicitly in integral form, and involves much less computation than in previous proofs of the result.
\end{abstract}

1. Introduction. In this paper we consider the nonselfadjoint singularly perturbed boundary value problem

$$
\begin{gathered}
L u \equiv \varepsilon u^{\prime \prime}+a u^{\prime}=f \quad \text { on }(0,1), \\
u(0)=u_{0}, \quad u(1)=u_{1},
\end{gathered}
$$

where the functions $a$ and $f$ are in $C^{2}[0,1], a(x) \geqslant \alpha>0$ on $[0,1], \varepsilon$ is a parameter in $(0,1], a$ and $f$ do not depend on $\varepsilon$, and $u_{0}, u_{1}$ are fixed constants. Under these assumptions, (1.1) has a unique solution $u(x)$. This solution has, in general, a boundary layer at $x=0$ for $\varepsilon$ near 0 .

A difference scheme for solving (1.1) on a uniform mesh in $[0,1]$ was proposed in El-Mistikawy and Werle [2]. In Berger et al. [1] and Hegarty et al. [4] two independent proofs were given that the El-Mistikawy and Werle scheme was uniformly second-order accurate (that is, all nodal errors are bounded by $C h^{2}$, where the constant $C$ is independent of $x, h$ and $\varepsilon$ ). Although these proofs differ greatly in their details, both use finite-difference techniques and involve large amounts of computation and estimation.

We give below a new proof of the uniform second-order accuracy of the El-Mistikawy and Werle scheme. An outline of the proof was given in Stynes and O'Riordan [11]. It has previously been shown by O'Riordan [8], [9] that a certain choice of finite elements together with a nonstandard quadrature rule generate the scheme. Thus the problem of proving the accuracy of the scheme can be approached from a finite-element viewpoint. The key to the proof is the introduction of a "discretized Green's function" associated with a modified version of (1.1), obtained by replacing the functions $a$ and $f$ by piecewise constant approximations. The nodal errors are then easily expressed explicitly as integrals involving the discretized

Received June 28, 1984; revised March 5, 1985.

1980 Mathematics Subject Classification. Primary 65L10; Secondary 34B27, 34E15.

*This paper was written while both authors were at Waterford Regional Technical College, Waterford, Ireland. 
Green's function (see Section 4). Most of these integrals are seen almost immediately to be bounded by $C h^{2}$, and we are left with the problem of estimating a single integral involving the boundary-layer term from an asymptotic expansion of $u$. Some computation is necessary to bound this integral, but it is very much less than the difficulties involved in the finite-difference proofs of the result.

With a natural choice of trial functions the Petrov-Galerkin finite-element method used here is globally uniformly first-order accurate, as shown in O'Riordan [8], [9]. Thus the bound proven here is a superconvergence result.

The discretized Green's function technique can readily be applied to other singularly perturbed problems. In [12] we use it to prove that a certain difference scheme is uniformly second-order accurate for a conservative nonselfadjoint singularly perturbed two-point boundary value problem. In fact, as may be seen in [12], the discretized Green's function actually suggests (indirectly) a good choice of difference scheme for this problem.

2. Finite-Element Generation of the El-Mistikawy and Werle Scheme. Let $N$ be a positive integer and let $h=1 / N$ be the uniform mesh width. The nodes in $[0,1]$ are $x_{i}=i h, i=0,1, \ldots, N$.

Define the piecewise constant approximation $\bar{a}(x)$ of $a(x)$ on $[0,1]$ by

$$
\bar{a}(x)= \begin{cases}\bar{a}_{i}, & x \in\left[x_{i-1}, x_{i}\right), i=1, \ldots, N-1, \\ \bar{a}_{N}, & x \in\left[x_{N-1}, x_{N}\right],\end{cases}
$$

where

$$
\bar{a}_{j}=\left(a\left(x_{j-1}\right)+a\left(x_{j}\right)\right) / 2 \text { for } j=1, \ldots, N .
$$

We then take our trial functions $\left\{\phi_{i}\right\}_{i=0}^{N}$ to satisfy

$$
\begin{gathered}
\bar{L} \phi_{i} \equiv \varepsilon \phi_{i}^{\prime \prime}+\bar{a} \phi_{i}^{\prime}=0 \quad \text { on }\left(x_{j}, x_{j+1}\right) \text { for } j=0, \ldots, N-1, \\
\phi_{i}\left(x_{j}\right)=\delta_{i, j} \quad \text { for } j=0, \ldots, N,
\end{gathered}
$$

where $\delta_{i, j}$ is the Kronecker delta. Thus each $\phi_{i}$ has its support in one or two subintervals.

The test functions $\left\{\psi_{k}\right\}_{k=1}^{N-1}$ are chosen differently. They are each defined by

$$
\begin{gathered}
\varepsilon \psi_{k}^{\prime \prime}-\bar{a} \psi_{k}^{\prime}=0 \text { on }\left(x_{j}, x_{j+1}\right) \text { for } j=0, \ldots, N-1, \\
\psi_{k}\left(x_{j}\right)=\delta_{k, j} \text { for } j=0, \ldots, N .
\end{gathered}
$$

Each $\psi_{k}$ has support $\left[x_{k-1}, x_{k+1}\right]$. These test functions were first introduced by Hemker [5]. A number of error estimates obtained using them were presented in de Groen and Hemker [3].

Define the trial space $S^{h}$ to be the span of the $\left\{\phi_{i}\right\}$, and the test space $T^{h}$ to be the span of the $\left\{\psi_{k}\right\}$. The Petrov-Galerkin approximation in $S^{h}$ to $u(x)$ is

$$
u^{h}(x) \equiv \sum_{i=0}^{N} u^{h}\left(x_{i}\right) \phi_{i}(x), \quad x \in[0,1]
$$

The $u^{h}\left(x_{i}\right)$ are determined in principle from the weak formulation

$$
\begin{aligned}
B_{\varepsilon}\left(u^{h}, \psi\right) & =(f, \psi) \quad \text { for all } \psi \in T^{h}, \\
u^{h}(0) & =u_{0}, \quad u^{h}(1)=u_{1},
\end{aligned}
$$


where

$$
B_{\varepsilon}(v, w) \equiv\left(v^{\prime},-\varepsilon w^{\prime}+a w\right), \quad v, w \in H^{1}(0,1)
$$

and

$$
(v, w)=\int_{0}^{1} v(x) w(x) d x, \quad v, w \in L^{2}(0,1) .
$$

However, the integrals in (2.2) cannot in general be evaluated exactly, so some quadrature rule must be employed. Following O'Riordan [9] we replace the functions $a$ and $f$ by $\bar{a}$ and $\bar{f}$ respectively, where $\bar{f}$ is defined analogously to $\bar{a}$. The integrals can then be evaluated exactly. We now have an approximation $\bar{u}^{h}(x) \in S^{h}$ to $u(x)$ with

$$
\bar{u}^{h}(x)=\sum_{i=0}^{N} \bar{u}^{h}\left(x_{i}\right) \phi_{i}(x), \quad x \in[0,1] .
$$

The $\bar{u}^{h}\left(x_{i}\right)$ are determined from

$$
\begin{array}{rlrl}
\bar{B}_{\varepsilon}\left(\bar{u}^{h}, \psi\right) & =(\bar{f}, \psi) & \text { for all } \psi \in T^{h}, \\
\bar{u}^{h}(0) & =u_{0}, \quad \bar{u}^{h}(1)=u_{1},
\end{array}
$$

where

$$
\bar{B}_{\varepsilon}(v, w) \equiv\left(v^{\prime},-\varepsilon w^{\prime}+\bar{a} w\right), \quad v, w \in H^{1}(0,1) .
$$

On evaluating (2.3) explicitly, one obtains the El-Mistikawy and Werle difference scheme for the $\bar{u}^{h}\left(x_{i}\right)$, as shown in O'Riordan [8], [9]. This calculation is easy but tedious, so we do not reproduce it here. It is interesting to note that the difference scheme generated does not depend on the specific choice of $\phi_{i}$ made above; one merely needs the usual trial function properties that each $\phi_{i}$ has support $\left[x_{i-1}, x_{i+1}\right]$ with $\phi_{i}\left(x_{j}\right)=\delta_{i, j}$.

For our choice of $S^{h}$ and $T^{h}$, it is shown in O'Riordan [9] that $\left\|u-\bar{u}^{h}\right\|_{\infty} \leqslant C h$ (here and throughout the paper $C$ denotes a generic constant independent of $x, h$ and $\varepsilon$ ). Thus, the bound we prove below, that

$$
\max _{0 \leqslant i \leqslant N}\left|u\left(x_{i}\right)-\bar{u}^{h}\left(x_{i}\right)\right| \leqslant C h^{2},
$$

is a superconvergence result.

3. Discretized Green's Function. For each $j \in\{1, \ldots, N-1\}$ we define a discretized Green's function $G_{j}$. Formally it satisfies

$$
\bar{L}^{T} G_{j}(x) \equiv \varepsilon G_{j}^{\prime \prime}(x)-\left(\bar{a}(x) G_{j}(x)\right)^{\prime}=\delta\left(x-x_{j}\right), \quad G_{j}(0)=G_{j}(1)=0,
$$

where $\delta(\cdot)$ is the Dirac $\delta$-distribution.

More precisely, $G_{j}$ is defined by

(3.1a) $G_{j} \in C[0,1]$,

(3.1b) $G_{j}(0)=G_{j}(1)=0$,

(3.1c) $\quad G_{j}^{\prime \prime}$ exists and is continuous on $[0,1]^{\wedge}$, where $[0,1]^{\wedge}$

$$
\text { denotes }[0,1] \backslash\left\{x_{1}, \ldots, x_{N-1}\right\} \text {, }
$$

(3.1d) $\varepsilon G_{j}^{\prime \prime}-\bar{a} G_{j}^{\prime}=0$ on $[0,1]^{\wedge}$,

(3.1e) $\quad \lim _{x \rightarrow x_{i}^{-}}\left(\varepsilon G_{j}^{\prime}-\bar{a} G_{j}\right)-\lim _{x \rightarrow x_{i}^{+}}\left(\varepsilon G_{j}^{\prime}-\bar{a} G_{j}\right)=-\delta_{i, j} \quad$ for $i=1, \ldots, N-1$. 
Remark. (3.1e) is equivalent to

$$
\varepsilon G_{j}^{\prime}(x)-\bar{a}(x) G_{j}(x)= \begin{cases}d & \text { on }\left[0, x_{j}\right) \cap[0,1]^{\wedge}, \\ d+1 & \text { on }\left(x_{j}, 1\right] \cap[0,1]^{\wedge},\end{cases}
$$

where $d=d(j, h, \varepsilon)$. Setting $x=0$ gives $d=\varepsilon G_{j}^{\prime}(0)$.

Notation. $\rho_{\alpha} \equiv \alpha h / \varepsilon, \rho_{i} \equiv \bar{a}_{i} h / \varepsilon$ for $i=1, \ldots, N$.

We note that Lemmas 3.1 and 3.2 are in fact valid for any choice of $\bar{a}_{i} \geqslant \alpha$, $i=1, \ldots, N$.

LEMMA 3.1. $G_{j} \in T^{h}$.

Proof. We must show that constants $\alpha_{k}$ can be chosen such that

$$
G_{j}=\sum_{k=1}^{N-1} \alpha_{k} \psi_{k}
$$

Clearly, (3.1a), (3.1b), (3.1c) and (3.1d) are satisfied for any choice of $\left\{\alpha_{k}\right\}$. Using integration by parts on $\left[x_{i-1}, x_{i}\right]$ and $\left[x_{i}, x_{i+1}\right]$ to evaluate $\bar{B}_{\varepsilon}\left(\phi_{i}, G_{j}\right)$, one sees from (3.1e) that the $\left\{\alpha_{k}\right\}$ must satisfy

$$
\sum_{k=1}^{N-1} \alpha_{k} \bar{B}_{\varepsilon}\left(\phi_{i}, \psi_{k}\right)=\delta_{i, j} \quad \text { for } i=1, \ldots, N-1 .
$$

But (2.3) may be written as

$$
\sum_{i=1}^{N-1} \bar{u}^{h}\left(x_{i}\right) \bar{B}_{\varepsilon}\left(\phi_{i}, \psi_{k}\right)=\left(\bar{f}, \psi_{k}\right) \quad \text { for } k=1, \ldots, N-1 .
$$

Hence, the matrix of the linear system of equations (3.3) is the transpose of the matrix of the linear system (3.4). This latter matrix is the matrix of the El-Mistikawy and Werle difference scheme, which is well known to be invertible (see, e.g., Berger et al. [1]). Consequently the matrix of (3.3) is invertible, and we are done.

LeMma 3.2. Let $\bar{A}(x)=\int_{0}^{x} \bar{a}(t) d t$ for $0 \leqslant x \leqslant 1$. Then

(i)

$$
G_{j}(x)= \begin{cases}d \varepsilon^{-1} \exp (\bar{A}(x) / \varepsilon) \int_{0}^{x} \exp (-\bar{A}(t) / \varepsilon) d t, & 0 \leqslant x \leqslant x_{j}, \\ -(d+1) \varepsilon^{-1} \exp (\bar{A}(x) / \varepsilon) \int_{x}^{1} \exp (-\bar{A}(t) / \varepsilon) d t, & x_{j} \leqslant x \leqslant 1\end{cases}
$$

(ii)

$$
d=\frac{-\int_{x}^{1}, \exp (-\bar{A}(t) / \varepsilon) d t}{\int_{0}^{1} \exp (-\bar{A}(t) / \varepsilon) d t}
$$

(iii) $G_{j}<0$ on $(0,1)$.

(iv) $G_{j}$ is strictly decreasing on $\left[0, x_{j}\right]$.

(v) $G_{j}>-1 / \alpha$ on $[0,1]$.

Proof. Multiplying (3.2) by the integrating factor $\varepsilon^{-1} \exp (-\bar{A}(x) / \varepsilon)$ and integrating from 0 to $x$ (for $x \leqslant x_{j}$ ) or from $x$ to 1 (for $x \geqslant x_{j}$ ) yields (i).

Equating the two formulas of (i) at $x=x_{j}$ gives (ii).

From (ii), $-1<d<0$, and then (i) yields (iii) immediately. 
Note that $\left(G_{j}(x) \exp (-\bar{A}(x) / \varepsilon)\right)^{\prime}=d \varepsilon^{-1} \exp (-\bar{A}(x) / \varepsilon)$ on $\left[0, x_{j}\right] \cap[0,1] \hat{~}$. Hence, $G_{j}(x) \exp (-\bar{A}(x) / \varepsilon)$ is strictly decreasing on $\left[0, x_{j}\right]$. But on $\left(0, x_{j}\right), G_{j}<0$ and $\exp (-\bar{A}(x) / \varepsilon)>0$ is strictly decreasing. This implies (iv).

For (v) suppose that $G_{j}(z) \leqslant-1 / \alpha$, where $z \in\left[x_{i-1}, x_{i}\right]$ for some $i$. Then (with appropriate modifications if $z$ is a node)

$$
\begin{aligned}
\varepsilon G_{j}^{\prime}(z) & =\bar{a}_{i} G_{j}(z)+ \begin{cases}d & \text { if } i \leqslant j, \\
d+1 & \text { if } i>j\end{cases} \\
& \leqslant d \quad(\text { by choice of } z) \\
& <0 .
\end{aligned}
$$

It follows that $G_{j}$ is strictly decreasing on $\left[z, x_{i}\right]$. Hence, $G_{j}\left(x_{i}\right) \leqslant-1 / \alpha$, and the above argument can be repeated on successive intervals until we obtain $G_{j}(1) \leqslant-1 / \alpha$, contradicting $G_{j}(1)=0$.

The next lemma is a technical result needed only to prove Lemma 3.4. Both of these lemmas remain valid for any choice of $\bar{a}$ satisfying $\bar{a}_{i} \geqslant \alpha$ and $\left|\bar{a}_{i}-\bar{a}_{i-1}\right| \leqslant C h$, $i=1, \ldots, N$.

LEMMA 3.3. For $j \leqslant i \leqslant N$,

$$
\left|\bar{a}_{i} G_{j}\left(x_{i}\right)+d+1\right| \leqslant C\left(\exp \left(-(N-i) \rho_{\alpha}\right)+h \sum_{k=0}^{N-i-1} \exp \left(-k \rho_{\alpha}\right)\right) \text {. }
$$

Proof. For $j<i \leqslant N$,

$$
\varepsilon G_{j}^{\prime}(x)-\bar{a}_{i} G_{j}(x)=d+1 \text { on }\left(x_{i-1}, x_{i}\right) .
$$

Thus,

$$
\left(G_{j}(x) \exp \left(-\bar{a}_{i} x / \varepsilon\right)\right)^{\prime}=\varepsilon^{-1}(d+1) \exp \left(-\bar{a}_{i} x / \varepsilon\right) \text { on }\left(x_{i-1}, x_{i}\right) .
$$

Integrating this from $x=x_{i-1}$ to $x=x_{i}$ and dividing by $\exp \left(-\bar{a}_{i} x_{i-1} / \varepsilon\right)$ yields

$$
G_{j}\left(x_{i}\right) \exp \left(-\rho_{i}\right)-G_{j}\left(x_{i-1}\right)=(d+1)\left(1-\exp \left(-\rho_{i}\right)\right) / \bar{a}_{i} .
$$

We now use induction on $i$ to prove the lemma.

For $i=N$ the lemma holds since $|d+1|<1$ by Lemma 3.2(ii).

Assume that the lemma holds for some $i$ with $j<i \leqslant N$. We deduce that it also holds for $i-1$ :

$$
\begin{aligned}
\bar{a}_{i-1} G_{j}\left(x_{i-1}\right) & +d+1=\left(\bar{a}_{i-1}-\bar{a}_{i}\right) G_{j}\left(x_{i-1}\right)+\bar{a}_{i} G_{j}\left(x_{i-1}\right)+d+1 \\
& =\left(\bar{a}_{i-1}-\bar{a}_{i}\right) G_{j}\left(x_{i-1}\right)+\left(\bar{a}_{i} G_{j}\left(x_{i}\right)+d+1\right) \exp \left(-\rho_{i}\right), \quad \text { by }(3.6) .
\end{aligned}
$$

By the inductive hypothesis and Lemma 3.2, parts (iii) and (v)

$$
\begin{aligned}
& \left|a_{i-1} G_{j}\left(x_{i-1}\right)+d+1\right| \\
& \quad \leqslant C\left(h+\exp \left(-\rho_{\alpha}\right)\left(\exp \left(-(N-i) \rho_{\alpha}\right)+h \sum_{k=0}^{N-i-1} \exp \left(-k \rho_{\alpha}\right)\right)\right) \\
& \quad=C\left(\exp \left(-(N-i+1) \rho_{\alpha}\right)+h \sum_{k=0}^{N-i} \exp \left(-k \rho_{\alpha}\right)\right)
\end{aligned}
$$

as required. 
Lemma 3.4. $\int_{0}^{1}\left|G_{j}^{\prime}(x)\right| d x \leqslant C$.

Proof.

$$
\int_{0}^{1}\left|G_{j}^{\prime}(x)\right| d x=\int_{0}^{x_{j}}\left|G_{j}^{\prime}(x)\right| d x+\sum_{i=j+1}^{N} \int_{x_{i-1}}^{x_{i}}\left|G_{j}^{\prime}(x)\right| d x .
$$

Now $\int_{0}^{x_{j}}\left|G_{j}^{\prime}(x)\right| d x=-G_{j}\left(x_{j}\right) \leqslant C$ by Lemma 3.2. For the other term, integrate (3.5) from $x \in\left(x_{i-1}, x_{i}\right)$ to $x_{i}$, where $i>j$. This gives

$$
\begin{aligned}
& G_{j}\left(x_{i}\right) \exp \left(-\bar{a}_{i} x_{i} / \varepsilon\right)-G_{j}(x) \exp \left(-\bar{a}_{i} x / \varepsilon\right) \\
& \quad=(d+1)\left(\exp \left(-\bar{a}_{i} x / \varepsilon\right)-\exp \left(-\bar{a}_{i} x_{i} / \varepsilon\right)\right) / \bar{a}_{i} .
\end{aligned}
$$

Solving for $G_{j}(x)$, then differentiating, gives

$$
G_{j}^{\prime}(x)=\varepsilon^{-1} \exp \left(-\bar{a}_{i}\left(x_{i}-x\right) / \varepsilon\right)\left(\bar{a}_{i} G_{j}\left(x_{i}\right)+d+1\right) .
$$

Thus

$$
\begin{aligned}
& \int_{x_{i-1}}^{x_{i}}\left|G_{j}^{\prime}(x)\right| d x=\left(1-\exp \left(-\rho_{i}\right)\right)\left|\bar{a}_{i} G_{j}\left(x_{i}\right)+d+1\right| / \bar{a}_{i} \\
& \leqslant C\left(1-\exp \left(-\rho_{\beta}\right)\right)\left(\exp \left(-(N-i) \rho_{\alpha}\right)+h \sum_{k=0}^{N-i-1} \exp \left(-k \rho_{\alpha}\right)\right) \\
& \text { by Lemma 3.3, where } \rho_{\beta}=(h / \varepsilon) \max _{[0,1]} a(x), \\
&=C\left(1-\exp \left(-\rho_{\beta}\right)\right) \\
& \quad \times\left\{\exp \left(-(N-i) \rho_{\alpha}\right)+h\left(1-\exp \left(-(N-i) \rho_{\alpha}\right)\right) /\left(1-\exp \left(-\rho_{\alpha}\right)\right)\right\} \\
& \leqslant C\left\{\left(1-\exp \left(-\rho_{\beta}\right)\right) \exp \left(-(N-i) \rho_{\alpha}\right)+h\right\},
\end{aligned}
$$

since $\left(1-\exp \left(-\rho_{\beta}\right)\right) /\left(1-\exp \left(-\rho_{\alpha}\right)\right) \leqslant \beta / \alpha$ holds for $0<\alpha \leqslant \beta$, as may be easily shown using elementary calculus. Now, summing over $i$ yields

$$
\begin{aligned}
& \sum_{i=j+1}^{N} \int_{x_{i-1}}^{x_{i}}\left|G_{j}^{\prime}(x)\right| d x \\
& \quad \leqslant C\left\{\left(1-\exp \left(-\rho_{\beta}\right)\right)\left(1-\exp \left(-(N-j) \rho_{\alpha}\right)\right) /\left(1-\exp \left(-\rho_{\alpha}\right)\right)+1\right\} \\
& \quad \leqslant C,
\end{aligned}
$$

which completes the proof.

4. A Formula for the Nodal Error. For any $j \in\{1, \ldots, N-1\}$ the nodal error at $x_{j}$ is

$$
\begin{aligned}
u\left(x_{j}\right)-\bar{u}^{h}\left(x_{j}\right) & =\left(\left(u-\bar{u}^{h}\right)(x), \delta\left(x-x_{j}\right)\right) \\
& =\left(\left(u-\bar{u}^{h}\right)(x), \bar{L}^{T} G_{j}\right)=\bar{B}_{\varepsilon}\left(u, G_{j}\right)-\bar{B}_{\varepsilon}\left(\bar{u}^{h}, G_{j}\right) .
\end{aligned}
$$

Now

$$
\begin{aligned}
\bar{B}_{\varepsilon}\left(\bar{u}^{h}, G_{j}\right) & =\left(\bar{f}, G_{j}\right), \quad \text { by }(2.3) \text { and Lemma } 3.1, \\
& =\left(f, G_{j}\right)+\left(\bar{f}-f, G_{j}\right)=\left(L u, G_{j}\right)+\left(\bar{f}-f, G_{j}\right) \\
& =B_{\varepsilon}\left(u, G_{j}\right)+\left(\bar{f}-f, G_{j}\right), \quad \text { integrating by parts. }
\end{aligned}
$$


Hence, (4.1) becomes

$$
\begin{aligned}
u\left(x_{j}\right)-\bar{u}^{h}\left(x_{j}\right) & =\bar{B}_{\varepsilon}\left(u, G_{j}\right)-B_{\varepsilon}\left(u, G_{j}\right)+\left(f-\bar{f}, G_{j}\right) \\
& =\left(u^{\prime},(\bar{a}-a) G_{j}\right)+\left(f-\bar{f}, G_{j}\right) .
\end{aligned}
$$

5. Estimation of the Nodal Error. Our approach here is to replace $u$ in the right-hand side of (4.2) by an asymptotic expansion. Each of the resulting terms is then shown to be bounded by $C h^{2}$.

LEMMA 5.1. Let $r \in C^{2}[0,1]$ be independent of $\varepsilon$. Let $s \in C[0,1]$ with $s \in$ $C^{1}\left(x_{i}, x_{i+1}\right)$ for each $i$ and $\int_{0}^{1}\left|s^{\prime}(t)\right| d t$ defined. Define $\bar{r}$ (piecewise constant approximation of $r)$ on $[0,1]$ analogously to the definitions of $\bar{a}$ and $\bar{f}$. Then

$$
|(r-\bar{r}, s)| \leqslant C h^{2}\left\{|s(1)|+\int_{0}^{1}\left|s^{\prime}(t)\right| d t\right\} .
$$

Proof. For $0 \leqslant x \leqslant 1$ set $R(x)=\int_{0}^{x} r(t) d t, \bar{R}(x)=\int_{0}^{x} \bar{r}(t) d t$. Then the classical error estimate for the trapezoidal rule gives

$$
\left|R\left(x_{i}\right)-\bar{R}\left(x_{i}\right)\right| \leqslant C h^{2} x_{i} \text { for } i=0, \ldots, N .
$$

For any $x$ in $[0,1]$ we have $x \in\left[x_{i}, x_{i+1}\right]$ for some $i$. Hence,

$$
\begin{aligned}
|R(x)-\bar{R}(x)| & =\left|R\left(x_{i}\right)-\bar{R}\left(x_{i}\right)-\int_{x_{i}}^{x}(\bar{r}(t)-r(t)) d t\right| \\
& \leqslant C h^{2} x_{i}+C h^{2}, \quad \text { as }|\bar{r}-r| \leqslant C h, \\
& \leqslant C h^{2} .
\end{aligned}
$$

Thus, integrating by parts,

$$
\begin{aligned}
|(r-\bar{r}, s)| & =\left|(R(1)-\bar{R}(1)) s(1)-\int_{0}^{1}(R(t)-\bar{R}(t)) s^{\prime}(t) d t\right| \\
& \leqslant C h^{2}\left\{|s(1)|+\int_{0}^{1}\left|s^{\prime}(t)\right| d t\right\} .
\end{aligned}
$$

Corollary 5.2. $\left|\left(f-\bar{f}, G_{j}\right)\right| \leqslant C h^{2}$.

Proof. Use Lemma 3.4.

Notation. $O\left(h^{i}\right)$ denotes a quantity whose absolute value is bounded by $C h^{i}$, $i=0,1,2$.

Applying Corollary 5.2 to (4.2) gives

$$
u\left(x_{j}\right)-\bar{u}^{h}\left(x_{j}\right)=\left(u^{\prime},(\bar{a}-a) G_{j}\right)+O\left(h^{2}\right) .
$$

Lemma 5.3 (Berger et Al. [1, Lemma 3.2], SMith [10]).

$$
u(x)=B_{0}(x)+C(a(x))^{-1} \exp (-A(x) / \varepsilon)+\varepsilon R_{0}(x),
$$

where $B_{0}$ is smooth and independent of $\varepsilon$, and $A(x)=\int_{0}^{x} a(t) d t$ for $0 \leqslant x \leqslant 1$. The function $R_{0}$ satisfies

$$
\begin{aligned}
& L R_{0}(x)=F_{0}(x, \varepsilon) \quad \text { on }(0,1), \\
& R_{0}(0)=0, \quad R_{0}(1)=\gamma_{0}(\varepsilon),
\end{aligned}
$$

where for $\varepsilon \in(0,1],\left|\gamma_{0}(\varepsilon)\right| \leqslant C$ and $\left|F_{0}(x, \varepsilon)\right| \leqslant C$ for $0 \leqslant x \leqslant 1$. 
Remark. By Kellogg and Tsan [7],

$$
\left|R_{0}^{(i)}(x)\right| \leqslant C\left(1+\varepsilon^{-i} \exp \left(-C_{1} x / \varepsilon\right)\right) \text { for } 0 \leqslant x \leqslant 1, i=0,1,
$$

where $C_{1}>0$ is a constant independent of $x, h$ and $\varepsilon$. Hence $\int_{0}^{1}\left|\varepsilon R_{0}^{\prime \prime}(x)\right| d x=$ $\int_{0}^{1}\left|-a(x) R_{0}^{\prime}(x)+F_{0}(x, \varepsilon)\right| \leqslant C$.

From Lemma 5.3,

$$
u^{\prime}(x)=-C \varepsilon^{-1} \exp (-A(x) / \varepsilon)+J(x),
$$

where

$$
J(x)=B_{0}^{\prime}(x)+\varepsilon R_{0}^{\prime}(x)-C a^{\prime}(x)(a(x))^{-2} \exp (-A(x) / \varepsilon) .
$$

Substitute (5.4) into (5.1). Note that

$$
\int_{0}^{1}\left|\left(J G_{j}\right)^{\prime}(x)\right| d x \leqslant C
$$

using $\int_{0}^{1}\left|G_{j}^{\prime}\right| \leqslant C,\left|G_{j}\right| \leqslant C$, and the above remark. Hence, by Lemma 5.1, we have

$$
u\left(x_{j}\right)-\bar{u}^{h}\left(x_{j}\right)=C\left(\varepsilon^{-1} \exp (-A / \varepsilon),(\bar{a}-a) G_{j}\right)+O\left(h^{2}\right) .
$$

Lemma 5.1 is too crude for this last integral, which requires some care.

6. Estimation of $\left(\varepsilon^{-1} \exp (-A / \varepsilon),(\bar{a}-a) G_{j}\right)$.

$$
\begin{aligned}
&\left(\varepsilon^{-1} \exp (-A / \varepsilon),(\bar{a}-a) G_{j}\right) \\
&=\left(\varepsilon^{-1}(\bar{a}-a) \exp ((\bar{A}-A) / \varepsilon), \exp (-\bar{A} / \varepsilon) G_{j}\right) \\
&=-\left(\exp ((\bar{A}-A) / \varepsilon),\left(\exp (-\bar{A} / \varepsilon) G_{j}\right)^{\prime}\right), \quad \text { on integrating by parts, } \\
&=-\int_{0}^{x_{j}} d \varepsilon^{-1} \exp (-A(x) / \varepsilon) d x \\
&-\int_{x_{j}}^{1}(d+1) \varepsilon^{-1} \exp (-A(x) / \varepsilon) d x \quad \text { from }(3.2), \\
&=\left\{Z(1)\left(Z\left(x_{j}\right)-\bar{Z}\left(x_{j}\right)\right)-Z\left(x_{j}\right)(Z(1)-\bar{Z}(1))\right\} / \bar{Z}(1),
\end{aligned}
$$

by Lemma 3.2(ii), where for $0 \leqslant x \leqslant 1$,

$$
Z(x)=\varepsilon^{-1} \int_{0}^{x} \exp (-A(t) / \varepsilon) d t, \quad \bar{Z}(x)=\varepsilon^{-1} \int_{0}^{x} \exp (-\bar{A}(t) / \varepsilon) d t .
$$

Lemma 6.1. Let $x \in[0,1]$. Set

$$
\eta(x)=\bar{A}(x)-A(x) \text { and } \xi(x)=\varepsilon^{-2} \eta(x) \exp (-A(x) / \varepsilon) .
$$

Then

(i) $Z(x)-\bar{Z}(x)=\int_{0}^{x} \xi(t) d t+(h / \varepsilon)^{2} O\left(h^{2}\right)$.

(ii) $Z(1)-\bar{Z}(1)-Z\left(x_{j}\right)+\bar{Z}\left(x_{j}\right)=\int_{x_{j}}^{1} \xi(t) d t+O\left(h^{2}\right)$, for $j=1, \ldots, N-1$.

(iii) $|Z(x)-\bar{Z}(x)| \leqslant C h^{2} / \varepsilon$.

(iv) $Z(x)=(a(0))^{-1}-(a(x))^{-1} \exp (-A(x) / \varepsilon)+\varepsilon O(1)$.

Proof. (i)

$$
\begin{aligned}
\exp (-\bar{A}(x) / \varepsilon) & =\exp (-A(x) / \varepsilon) \exp (-\eta(x) / \varepsilon) \\
& =\exp (-A(x) / \varepsilon)\left\{1-\eta(x) / \varepsilon+\frac{1}{2}(\eta(x) / \varepsilon)^{2} \exp (\gamma / \varepsilon)\right\},
\end{aligned}
$$

where $\gamma=\gamma(x)$ is between 0 and $-\eta(x)$. 
Hence,

$$
\begin{aligned}
\exp (-A(x) / \varepsilon)-\exp (-\bar{A}(x) / \varepsilon) \\
\quad=(\eta(x) / \varepsilon) \exp (-A(x) / \varepsilon)-\frac{1}{2}(\eta(x) / \varepsilon)^{2} \exp (-\theta / \varepsilon),
\end{aligned}
$$

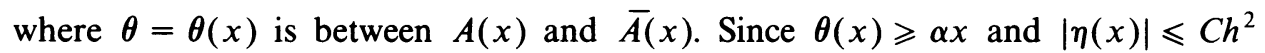
(classical trapezoidal rule error estimate), integrating (6.2) from 0 to $x$ yields (i).

(ii) Integrate (6.2) from $x_{j}$ to 1 , and use $(h / \varepsilon)^{2} \exp \left(-\alpha x_{j} / \varepsilon\right) \leqslant C$.

(iii) Taking one less term in the Taylor expansion above gives, instead of (6.2),

$$
\exp (-A(x) / \varepsilon)-\exp (-\bar{A}(x) / \varepsilon)=(\eta(x) / \varepsilon) \exp (-\omega / \varepsilon)
$$

where $\omega=\omega(x) \geqslant \alpha x$. Integrating this proves (iii).

(iv) Write $Z(x)$ as $\int_{0}^{x}-(a(t))^{-1}(-a(t) / \varepsilon) \exp (-A(t) / \varepsilon) d t$, integrate by parts, and use $-A(t) \leqslant-\alpha t$.

Applying Lemma 6.1, parts (iii) and (iv), to (6.1) yields

$$
\begin{aligned}
& \bar{Z}(1)\left(\varepsilon^{-1} \exp (-A / \varepsilon),(\bar{a}-a) G_{j}\right) \\
&=\left\{(a(0))^{-1}-(a(1))^{-1} \exp (-A(1) / \varepsilon)\right\}\left(Z\left(x_{j}\right)-\bar{Z}\left(x_{j}\right)\right) \\
&-\left\{(a(0))^{-1}-\left(a\left(x_{j}\right)\right)^{-1} \exp \left(-A\left(x_{j}\right) / \varepsilon\right)\right\}(Z(1)-\bar{Z}(1))+O\left(h^{2}\right) \\
&=\left(a\left(x_{j}\right)\right)^{-1} \exp \left(-A\left(x_{j}\right) / \varepsilon\right)(Z(1)-\bar{Z}(1)) \\
&-(a(0))^{-1}\left\{Z(1)-\bar{Z}(1)-Z\left(x_{j}\right)+\bar{Z}\left(x_{j}\right)\right\}+O\left(h^{2}\right) \\
&=\left(a\left(x_{j}\right)\right)^{-1} \exp \left(-A\left(x_{j}\right) / \varepsilon\right) \int_{0}^{1} \xi(x) d x \\
&-(a(0))^{-1} \int_{x_{j}}^{1} \xi(x) d x+O\left(h^{2}\right),
\end{aligned}
$$

by Lemma 6.1, parts (i) and (ii),

$$
\begin{aligned}
= & \left(a\left(x_{j}\right)\right)^{-1} \exp \left(-A\left(x_{j}\right) / \varepsilon\right) \int_{0}^{1-x_{j}} \xi(x) d x \\
& -(a(0))^{-1} \int_{0}^{1-x_{j}} \xi\left(x_{j}+x\right) d x+O\left(h^{2}\right),
\end{aligned}
$$

using $|\xi(x)| \leqslant C h^{2} \varepsilon^{-2} \exp (-\alpha x / \varepsilon)$.

LEMMA 6.2.

$$
\begin{aligned}
\int_{0}^{1-x_{j}} & \xi\left(x_{j}+x\right) d x \\
\quad= & \int_{0}^{1-x_{j}} \varepsilon^{-2} \eta\left(x_{j}+x\right) \exp \left\{-\left(A(x)+A\left(x_{j}\right)\right) / \varepsilon\right\} d x+O\left(h^{2}\right) .
\end{aligned}
$$

Proof. Let $\theta\left(x, x_{j}\right)=A\left(x+x_{j}\right)-A(x)-A\left(x_{j}\right)=\int_{0}^{x_{j}}(a(x+t)-a(t)) d t$, so $|\theta| \leqslant \int_{0}^{x_{j}} C x d t \leqslant C x x_{j}$. Now

$$
\begin{aligned}
\exp \left(-A\left(x+x_{j}\right) / \varepsilon\right) & =\exp \left\{-\left(A(x)+A\left(x_{j}\right)\right) / \varepsilon\right\} \exp (-\theta / \varepsilon) \\
& =\exp \left\{-\left(A(x)+A\left(x_{j}\right)\right) / \varepsilon\right\}-\theta \varepsilon^{-1} \exp (-D / \varepsilon),
\end{aligned}
$$


where $D=D\left(x, x_{j}\right)$ is between $A\left(x+x_{j}\right)$ and $A(x)+A\left(x_{j}\right)$, so $D \geqslant \alpha\left(x+x_{j}\right)$. Hence

$$
\begin{aligned}
\int_{0}^{1-x_{j}} \xi\left(x_{j}+x\right) d x= & \int_{0}^{1-x_{j}} \varepsilon^{-2} \eta\left(x_{j}+x\right) \exp \left\{-\left(A(x)+A\left(x_{j}\right)\right) / \varepsilon\right\} d x \\
& -\int_{0}^{1-x_{j}} \varepsilon^{-2} \eta\left(x_{j}+x\right) \theta \varepsilon^{-1} \exp (-D / \varepsilon) d x,
\end{aligned}
$$

and the last integral is bounded in absolute value by

$$
C h^{2}\left(x_{j} / \varepsilon\right) \exp \left(-\alpha x_{j} / \varepsilon\right) \int_{0}^{1-x_{j}}(x / \varepsilon) \exp (-\alpha x /(2 \varepsilon)) \varepsilon^{-1} \exp (-\alpha x /(2 \varepsilon)) d x \leqslant C h^{2} .
$$

Remark. Consequently, (6.3) becomes

$$
\begin{aligned}
& \bar{Z}(1)\left(\varepsilon^{-1} \exp (-A / \varepsilon),(\bar{a}-a) G_{j}\right) \\
&=\varepsilon^{-2} \exp \left(-A\left(x_{j}\right) / \varepsilon\right) \int_{0}^{1-x_{j}}\left\{\left(a\left(x_{j}\right)\right)^{-1} \eta(x)-(a(0))^{-1} \eta\left(x_{j}+x\right)\right\} \\
& \cdot \exp (-A(x) / \varepsilon) d x+O\left(h^{2}\right)
\end{aligned}
$$

Lemma 6.3. For $j \in\{1, \ldots, N-1\}$ and $0 \leqslant x \leqslant 1-x_{j}$,

$$
\left|(A-\bar{A})\left(x_{j}+x\right)-(A-\bar{A})(x)\right| \leqslant C h^{2} x_{j} .
$$

Proof. For $k=0, \ldots, N-j-1$, write $x_{k+1 / 2}$ for $\frac{1}{2}\left(x_{k}+x_{k+1}\right)$. For $x \in$ $\left[x_{k}, x_{k+1}\right)$, an easy Taylor expansion gives

$$
a(x)-\bar{a}(x)=\left(x-x_{k+1 / 2}\right) a^{\prime}\left(x_{k+1 / 2}\right)+O\left(h^{2}\right) .
$$

Now

$$
\begin{aligned}
& (A-\bar{A})\left(x_{j}+x\right)-(A-\bar{A})(x) \\
& \quad=\int_{x}^{x_{k+1}}(a-\bar{a})+\int_{x_{k+1}}^{x_{j+k}}(a-\bar{a})+\int_{x_{j+k}}^{x_{j}+x}(a-\bar{a}) .
\end{aligned}
$$

Here

$$
\left|\int_{x_{k+1}}^{x_{j+k}}(a-\bar{a})\right| \leqslant C h^{2} x_{j-1},
$$

by the classical trapezoidal rule. On the other hand,

$$
\begin{aligned}
\int_{x}^{x_{k+1}}(a-\bar{a})+\int_{x_{j+k}}^{x_{j}+x}(a-\bar{a}) \\
=\int_{x}^{x_{k+1}}\left(s-x_{k+1 / 2}\right) a^{\prime}\left(x_{k+1 / 2}\right) d s \\
\quad+\int_{x_{j+k}}^{x_{j}+x}\left(t-x_{j+k+1 / 2}\right) a^{\prime}\left(x_{j+k+1 / 2}\right) d t+O\left(h^{3}\right), \text { by }(6.5) .
\end{aligned}
$$


But $a^{\prime}\left(x_{j+k+1 / 2}\right)=a^{\prime}\left(x_{k+1 / 2}\right)+x_{j} a^{\prime \prime}(y)$, where $x_{k+1 / 2}<y<x_{j+k+1 / 2}$. Substituting this into (6.8), then letting $t=s+x_{j}$ to combine the $a^{\prime}\left(x_{k+1 / 2}\right)$ terms, yields

$$
\begin{gathered}
\int_{x_{k}}^{x_{k+1}}\left(s-x_{k+1 / 2}\right) a^{\prime}\left(x_{k+1 / 2}\right) d s+\int_{x_{k}}^{x}\left(s-x_{k+1 / 2}\right) x_{j} a^{\prime \prime}(y) d s+O\left(h^{3}\right) \\
=x_{j} O\left(h^{2}\right)+O\left(h^{3}\right) .
\end{gathered}
$$

Together with (6.6) and (6.7), this proves the lemma.

We now have, by Lemma 6.3,

$$
\begin{aligned}
& \left(a\left(x_{j}\right)\right)^{-1} \eta(x)-(a(0))^{-1} \eta\left(x_{j}+x\right) \\
& \quad=\left(a\left(x_{j}\right)\right)^{-1}(a(0))^{-1}\left\{\left(a(0)-a\left(x_{j}\right)\right) \eta(x)+a\left(x_{j}\right)\left(\eta(x)-\eta\left(x_{j}+x\right)\right)\right\} \\
& \quad=x_{j} O\left(h^{2}\right) .
\end{aligned}
$$

Consequently, from (6.4)

$$
\begin{aligned}
& \left|\bar{Z}(1)\left(\varepsilon^{-1} \exp (-A / \varepsilon),(\bar{a}-a) G_{j}\right)\right| \\
& \quad \leqslant C h^{2}\left(x_{j} / \varepsilon\right) \exp \left(-\alpha x_{j} / \varepsilon\right) \int_{0}^{1-x_{j}} \varepsilon^{-1} \exp (-\alpha x / \varepsilon) d x+O\left(h^{2}\right) \leqslant C h^{2} .
\end{aligned}
$$

It is easy to see that $\bar{Z}(1)$ is bounded below and above by positive constants independent of $h$ and $\varepsilon$. Thus, combining (5.5) and (6.9) proves

TheOrem 6.4. For $1 \leqslant j \leqslant N-1,\left|u\left(x_{j}\right)-\bar{u}^{h}\left(x_{j}\right)\right| \leqslant C h^{2}$.

Remark. The discretized Green's function technique can be used to give a quick proof that schemes similar to Il'in's [6] for (1.1) are $O(h)$. For example, if in Section 2 above we define $\bar{a}_{i}=a\left(x_{i}\right)$ and $\bar{f}_{i}=f\left(x_{i}\right), i=1, \ldots, N$, we only need Lemmas 3.1 and 3.2, Eq. (4.2), and $\int_{0}^{1}\left|u^{\prime}(x)\right| d x \leqslant C$ to get $O(h)$ nodal accuracy. In fact, the same simple argument proves $O(h)$ accuracy for any scheme generated using piecewise constant approximations $\bar{a}, \bar{f}$ of $a, f$ for which $\|a-\bar{a}\|_{\infty} \leqslant C h,\|f-\bar{f}\|_{\infty}$ $\leqslant C h$.

\section{Department of Science \\ Dundalk Regional Technical College \\ Dundalk, Ireland}

Department of Mathematics

University College

Cork, Ireland

1. A. E. Berger, J. M. Solomon \& M. Ciment, "An analysis of a uniformly accurate difference method for a singular perturbation problem," Math. Comp., v. 37, 1981, pp. 79-94.

2. T. M. EL-MistikaWY \& M. J. WeRLE, "Numerical method for boundary layers with blowing-the exponential box scheme," AIAA J., v. 16, 1978, pp. 749-751.

3. P. P. N. De Groen \& P. W. Hemker, "Error bounds for exponentially fitted Galerkin methods applied to stiff two-point boundary value problems," in Numerical Analysis of Singular Perturbation Problems (P. W. Hemker and J. J. H. Miller, eds.), Academic Press, New York, 1979, pp. 217-249.

4. A. F. Hegarty, J. J. H. Miller \& E. O'Riordan, “Uniform second order difference schemes for singular perturbation problems," in Boundary and Interior Layers-Computational and Asymptotic Methods (J. J. H. Miller, ed.), Boole Press, Dublin, 1980, pp. 301-305.

5. P. W. Hemker, A Numerical Study of Stiff Two-point Boundary Value Problems, Mathematical Centre, Amsterdam, 1977. 
6. A. M. IL'IN, "Differencing scheme for a differential equation with a small parameter affecting the highest derivative," Mat. Zametki, v. 6, 1969, pp. 237-248; English transl. in Math. Notes, v. 6, 1969. pp. 596-602.

7. R. B. Kellogg \& A. TSAN, "Analysis of some difference approximations for a singular perturbation problem without turning points," Math. Comp., v. 32, 1978, pp. 1025-1039.

8. E. O'Riordan, Finite Element Methods for Singularly Perturbed Problems, Ph. D. thesis, School of Mathematics, Trinity College, Dublin, 1982.

9. E. O'RioRDAN, "Singularly perturbed finite element methods," Numer. Math., v. 44, 1984, pp. 425-434.

10. D. R. SMIth, "The multivariable method in singular perturbation analysis," SIAM Rev., v. 17 , 1975, pp. 221-273.

11. M. StYNes \& E. O'RioRDAN, “A superconvergence result for a singularly perturbed boundary value problem," in BAIL III, Proc. Third International Conference on Boundary and Interior Layers (J. J. H. Miller, ed.), Boole Press, Dublin, 1984, pp. 309-313.

12. M. Stynes \& E. O'Riordan, "A uniformly accurate finite element method for a singular perturbation problem in conservative form," SIAMJ. Numer. Anal. (To appear.) 